\title{
A Theoretical Investigation of the Resonance Damping Performance of Magnetic Material Coating in Power/Ground Plane Structures
}

\author{
Lin-Kun Wu, Member, IEEE, and Chih-Hsiung Tseng
}

\begin{abstract}
Power bus structure, consisting of two parallel solid power and ground planes separated by an insulator, behaves as a cavity resonator at high frequencies. Noise on the power bus, due to a sudden change in the current drawn by an active component, can appear as an undesired spatial fluctuation in the voltage between power and ground, especially at resonant frequencies of the resultant cavity, which may lead to problems in signal integrity, excessive delays, false switching, and radiated emission. These resonances can be suppressed by introducing high-frequency loss into the structure. This paper investigates a simple method to reduce self-/transfer impedance of power/ground planes for mitigating power/ground bounce in high-speed printed circuit board design by adding a thin layer of magnetic material coating to the insidefacing surfaces of copper power and ground plates to increase their effective high-frequency surface impedance. The increased surface impedance will increase the attenuation constant of the propagating wave inside the cavity that benefits reduction of cavity's quality factor ( $Q$ factor). The simulation results obtained from a modified cavity resonator model show that increasing surface impedance can dramatically reduce self- and transfer impedances at board resonant frequencies.
\end{abstract}

Index Terms-Cavity resonator model, ground bounce, magnetic material coating, self-/transfer impedance, simultaneous switching noise (SSN), surface impedance.

\section{INTRODUCTION}

$\mathbf{I}$ $\mathrm{N}$ THE so-called simultaneous switching noise (SSN) or ground bounce problem, any sudden surge of the current drawn by active devices from power/ground planes at one point can cause voltage fluctuation at any other point across the entire printed circuit board (PCB) that is directly proportional to the transfer impedance between the two. As a closely spaced parallel plane pair forms a high- $Q$ cavity resonator, extremely high transfer impedance peaks can occur at resonance frequencies. With the operating frequency exceeding $1 \mathrm{GHz}$ and working voltages continuing to decrease for reducing power dissipation in high-speed digital circuits, noise in the power distribution system has become a major performance-limiting problem.

To avoid SSN or ground bounce, the power distribution systems are required to have low impedance across a broad bandwidth. Some commonly used mitigation techniques for control-

Manuscript received July 9, 2004; revised January 10, 2005 and May 17, 2005. This work was supported by the National Science Council, Taiwan, R.O.C., under Grant NSC93-2213-E-009-096.

The authors are with the Institute of Communication Engineering, National Chiao Tung University, Hsinchu, Taiwan 300, R.O.C. (e-mail: u8913806@cc.nctu.edu.tw).

Digital Object Identifier 10.1109/TEMC.2005.857362 ling board noise are: 1) added discrete decoupling capacitors between power and ground planes; 2) enhanced embedded capacitance (i.e., the natural capacitance between power and ground planes) to provide better decoupling [2]-[4]; 3) added dissipative termination along the board edges to reduce the resonance peaks [5]; and 4) added additional loss including dielectric, conduction, component, and/or radiation losses to damp board resonance [6], [12].

Discrete decoupling capacitors are employed to mitigate SSN noise in PCB design. Traditionally, equivalent series resistance (ESR) of decoupling capacitors is chosen to be small for providing low impedance at high frequency, but the existence of series inductance will reduce the effectiveness of decoupling capacitors. Their effective frequency range is limited by intrinsic inductance of capacitor and extrinsic inductance, including lead and interconnection (e.g., mounting pads, traces, and vias) inductances. At frequencies above its series resonance frequency, the increasingly higher inductive reactance defeats the purpose of low-impedance decoupling. Then, decoupling capacitors with small ESR will result in higher $Q$ factor and impedance peak at board resonant frequencies. In addition, use of a large number of decoupling capacitors consumes the valuable board area and reduces the reliability of $\mathrm{PCB}$.

$\mathrm{Xu}$ and Hubing exploited the concept of enhanced "embedded" capacitance by using a thin dielectric layer between the solid power and ground planes to effectively increase the influence of solid planes' conduction loss on damping board resonances [2]-[4]. If the spacing between the two solid planes is on the order of conductor's skin depth, conductor loss becomes quite effective in reducing resonant impedance peak. For this to happen, however, the substrate used needs to be extremely thin. This thin dielectric layer may present difficulty in board fabrication, for instance, vias through this thin layer may short the power and ground planes together.

Novak used dissipative edge termination to reduce board resonances [5]. Dissipative components placed along the edges of the board are designed to present load impedance matched to that of the impinging wave such that, ideally, no wave will be reflected back into the parallel-plate region. Without a reflection wave, the standing wave cannot be formed, and board resonance is thus prevented. Therefore, in principle, the board becomes an infinitely large parallel-plate radial waveguide. Because the component spacing has to be much smaller than the shortest wavelength of concern, however, a large number of dissipative components may be needed, which will limit its use in practical applications. 
To avoid transfer impedance peaks appearing at board resonant frequencies, we can introduce additional loss into the board to reduce its $Q$ factor. This may include increasing dielectric, conduction, component, and/or radiation losses. Generally, dielectric loss is kept small to provide good isolation and avoid leakage current between power and ground planes. The radiation loss from board edges, which behave as slot antennas, is proportional to the square of spacing between the two planes. In practice, the spacing is on the order of 10 mils on PCBs; therefore, radiation loss is minor compared with other losses and can be neglected.

More recently, decoupling capacitors with purposely increased ESR have also been proposed to suppress board resonances. Zeff and Hubing suggested applying such lossy components to reduce the impedance at board resonant frequencies [6]. Resistive components mounted between the power/ground pair can reduce the resulting cavity's $Q$ factor and impedance peak at resonant frequencies. Novak et al. suggested using the distributed matched bypassing (DMB) method to create a smooth impedance profile [12]. The DMB is implemented by annular resistive interstitial element screened-in (ARIES) that is based on the annular buried resistor (ABR). Printed resistors are inserted only at one of the capacitor terminals connecting the power plane for reducing the number of required components. The direct physical connection of the printed resistor can also decrease interconnection inductance.

In a design note, Johnson mentioned the idea of applying a thin coating of steel to the inside-facing surfaces of a power and ground plane pair to damp its resonances [7]. In this paper, a theoretical investigation of the potential performance of such a concept is reported. Surface impedance is incorporated into the well-known cavity model in Section II-A to model the effect of conductor loss. To increase high-frequency conductor loss, the two metallic layer structure constituted of a thin magnetic material coated on one side of the copper plate is then considered in Section II-B. For a better understanding of the relationship between the loss factor and impedance peak at resonance, the $Q$ factor of the cavity, including the effects of dielectric and conductor losses, is derived Section II-C. Theoretical results obtained for various coating thicknesses and material properties are used to evaluate the potential resonance damping performance of the proposed concept. Numerical results and discussions are presented in Section III, which is followed by conclusions in Section IV.

\section{Method of ANALYSIS}

\section{A. Cavity Resonator Model}

The rectangular parallel plane pair shown in Fig. 1 consists of two solid planes of the same size $a \times b$ separated by a dielectric of thickness $h$. It is assumed that dielectric thickness is much smaller than $a, b$ and the wavelength $(\lambda)$ of the highest frequency of interest. Under this assumption, radiation loss from board edges can be ignored. Therefore, this structure can be modeled as a $\mathrm{TM}_{z}$ cavity with two electric conductor planes representing the power and ground and each side of the rectangular board approximated as a perfect magnetic conductor. The ports $i$ and $j$

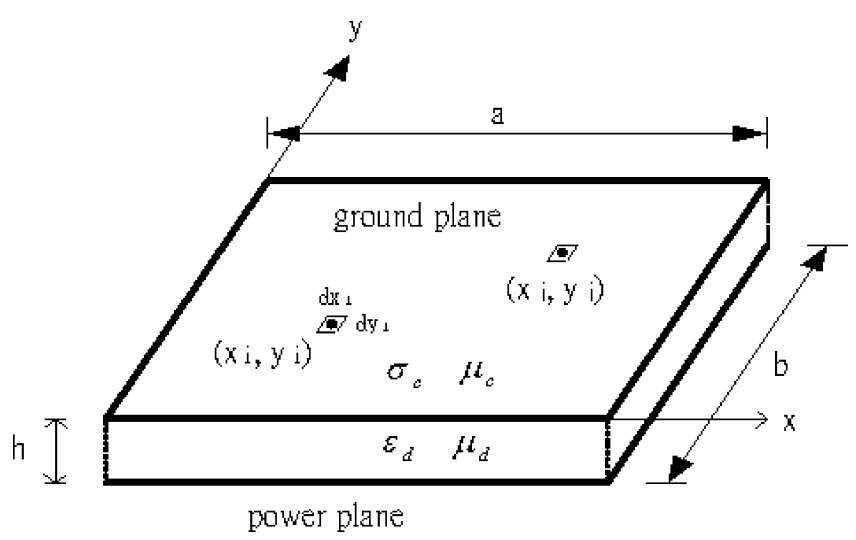

Fig. 1. Geometry of a rectangular power-ground plane pair.

are, respectively, located at $\left(x_{i}, y_{i}\right)$ and $\left(x_{j}, y_{j}\right)$ with rectangular cross section of size $d x_{i} \times d y_{i}$ and $d x_{j} \times d y_{j}$, which are much smaller than the shortest wavelength of interest. The transfer impedance is given by [1]-[5].

$$
\begin{aligned}
Z_{i j}= & j \omega \mu h \sum_{m=0}^{\infty} \sum_{n=0}^{\infty} \frac{\chi_{m n}^{2}}{a b\left(k_{x m}^{2}+k_{y n}^{2}+\gamma^{2}\right)} \\
& \times \cos \left(k_{x m} x_{i}\right) \cos \left(k_{y n} y_{i}\right) \\
& \times \operatorname{sinc}\left(k_{x m} \frac{d x_{i}}{2}\right) \operatorname{sinc}\left(k_{y n} \frac{d y_{i}}{2}\right) \\
& \times \cos \left(k_{x m} x_{j}\right) \cos \left(k_{y n} y_{j}\right) \\
& \times \operatorname{sinc}\left(k_{x m} \frac{d x_{j}}{2}\right) \operatorname{sinc}\left(k_{y n} \frac{d y_{j}}{2}\right) \\
k_{x m}= & \frac{m \pi}{a} \quad k_{y n}=\frac{n \pi}{b} \\
\chi_{m n}^{2}= & \begin{cases}1, & m=n=0 \\
2, & m=0 \text { or } n=0 \\
4, & m \neq 0 n \neq 0\end{cases}
\end{aligned}
$$

where $m$ and $n$ are indices of the cavity's $\mathrm{TM}_{z}$ modes. The complex propagation constant $\gamma$ of the parallel plane pair with the imperfect conductor and dielectric can be expressed as

$$
\gamma=j \omega \sqrt{\mu_{d} \varepsilon_{d}} \sqrt{\left[1-j \frac{2 Z_{s}}{\omega \mu_{d} h}\right] \cdot[1-j \tan \delta]}=\alpha+j \beta
$$

where

$$
\begin{aligned}
& \alpha=\omega \sqrt{\mu_{d} \varepsilon_{d}}\left(R^{2}+X^{2}\right)^{1 / 4} \sin \left[\frac{\tan ^{-1}\left(\frac{X}{R}\right)}{2}\right] \\
& \beta=\omega \sqrt{\mu_{d} \varepsilon_{d}}\left(R^{2}+X^{2}\right)^{1 / 4} \cos \left[\frac{\tan ^{-1}\left(\frac{X}{R}\right)}{2}\right] \\
& R=1+\frac{2 X_{s}}{\omega \mu_{d} h}-\frac{2 R_{s}}{\omega \mu_{d} h} \tan \delta \\
& X=\frac{2 R_{s}}{\omega \mu_{d} h}+\tan \delta+\frac{2 X_{s}}{\omega \mu_{d} h} \tan \delta .
\end{aligned}
$$


In (2), $R_{s}$ and $X_{s}$ are, respectively, the real and imaginary parts of the surface impedance $Z_{s}$ of the conductor, and $\mu_{d}, \varepsilon_{d}$, and $\tan \delta$ represent the permeability, permittivity, and loss tangent of the dielectric, respectively. For numerical calculation, the infinite summation must be truncated; in this study, the maximum $m$ and $n$ used are 1000, respectively, to achieve a 5\% maximum error at all resonant frequencies [2].

Because the imperfect dielectric and conductor introduce nonzero attenuation constant $\alpha$, the radial wave is thus attenuated as it propagates away from its source of excitation (i.e., the transient current flowing along the power/ground vias) and reflects back from the board edges. Therefore, coupling between different board locations is reduced, and the board's resonant behavior is suppressed. For a good dielectric and conductor [i.e., $\tan \delta, R_{s} /\left(\omega \mu_{d} h\right)$ and $X_{s} /\left(\omega \mu_{d} h\right) \ll 1$ and $R_{s} \approx X_{s}$ at high frequency], $R$ in (2d) approaches 1 , whereas $X$ in (2e) approaches $R_{s} /\left(\omega \mu_{d} h\right)+\tan \delta$, which increases as substrate thickness decreases. As a result, $\alpha$ increases when $h$ decreases; this concept has been presented in [2]-[4]. Alternatively, (2d) and (2e) also show that $R$ and $X$, and therefore $\alpha$, can be made larger by simply increasing the surface impedance of the conductor. This concept is explored further here.

\section{B. Surface Impedance of a Copper Plate Coated With a Thin Magnetic Material}

As mentioned previously, we can increase surface impedance to enhance attenuation constant for suppressing board resonances. To increase surface impedance, the inside-facing surfaces of the copper power and ground plates are each coated with a thin layer of magnetic material. The surface impedance of the composite two metallic layer structure is derived first.

Fig. 2 shows that an infinite plane conductor with conductivity $\sigma_{c 1}$, permeability $\mu_{c 1}$, and thickness $d_{1}$ is coated over another conductor of conductivity $\sigma_{c 2}$, permeability $\mu_{c 2}$, and thickness $d_{2}$, which is backed by a FR-4 substrate of infinite thickness. For a $\mathrm{TM}_{z}$ wave between the parallel-plate conductors with a very large but finite conductivity, the electromagnetic field will penetrate conductor and incur power loss in the plates [13]. This necessitates the presence of a nonvanishing axial electric field at the plate surfaces, and therefore, the penetrating field can be regarded as a plane wave normally incident onto each conductor plate. Then, the surface impedance of an imperfect conductor, which is the ratio of axial electric field to surface current density accompanying the transverse magnetic field, can also be treated as the input impedance looking into the conductor plate. Considering a plane wave normally incident onto the lower side of conductor 1 , the effective surface impedance of the composite structure can be obtained as $Z_{\text {in,1 }}$ from the equivalent transmission line model shown in Fig. 2 in which characteristic impedances $Z_{0,1}$ and $Z_{0,2}$ are the same as the intrinsic impedance of each of the two conductors (i.e., $\eta_{c, 1}$ and $\eta_{c, 2}$ ). From transmission line theory, conductor 2 can be treated as a transmission line of length $d_{2}$, and the input impedance $Z_{\text {in,2 }}$ looking into conductor 2 from conductor 1 is expressed as

$$
Z_{\text {in }, 2}=Z_{0,2} \frac{Z_{L}+Z_{0,2} \tanh \left(\gamma_{2} d_{2}\right)}{Z_{0,2}+Z_{L} \tanh \left(\gamma_{2} d_{2}\right)}
$$

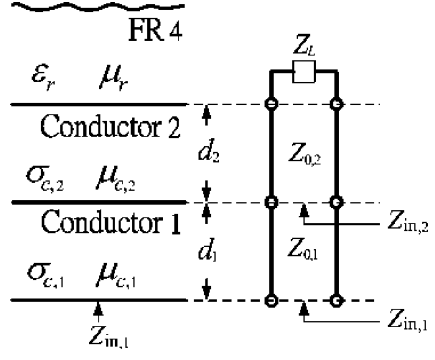

Fig. 2. Side view of the two metallic layer structure and its equivalent transmission line model.

where $\gamma_{2}$ is the complex propagation constant of the propagating wave within conductor 2 , and load impedance $Z_{L}$ is equal to the intrinsic impedance $\eta_{d}$ of the FR-4 substrate. Repeating this for the conductor 1 with $Z_{\mathrm{in}, 2}$ serves as its load impedance, and the desired input impedance or the surface impedance of the composite structure can then be derived as

$$
Z_{s}=Z_{0,1} \frac{Z_{\mathrm{in}, 2}+Z_{0,1} \tanh \left(\gamma_{1} d_{1}\right)}{Z_{0,1}+Z_{\mathrm{in}, 2} \tanh \left(\gamma_{1} d_{1}\right)}
$$

For the good conductor considered here, the various characteristic or intrinsic impedances and complex propagation constants appearing in (3) and (4) are given by

$$
\begin{gathered}
Z_{0, i}=\eta_{c, i}=(1+j) \sqrt{\frac{\pi f \mu_{c, i}}{\sigma_{c, i}}}, \quad i=1,2 \\
\gamma_{i}=(1+j) \sqrt{\pi f \mu_{c, i} \sigma_{c, i}}, \quad i=1,2 .
\end{gathered}
$$

When the frequency approaches 0 - and $\infty-\mathrm{Hz},(4)$ reduces to

$$
Z_{s}= \begin{cases}\frac{1}{\frac{1}{\eta_{d}}+\sigma_{c, 1} d_{1}+\sigma_{c, 2} d_{2}} & \left(\delta_{1}>>d_{1} \text { and } \delta_{2}>>d_{2}\right) \\ (1+j) \sqrt{\frac{\pi f \mu_{c, 1}}{\sigma_{c, 1}}} & \left(\delta_{1} \ll d_{1}\right)\end{cases}
$$

where $\delta_{i}$ is the skin depth, which is inversely proportional to the square root of frequency, of the $i$ th $(i=1$ or 2$)$ conductor. In (7a), the surface impedance of the composite structure is approximated as parallel connections of three resistors (i.e., each of the two conductor's dc sheet resistance and intrinsic impedance of the FR-4 substrate). At low frequencies, with $\eta_{d}$ being around $180 \Omega$ or FR-4 and the two $\sigma_{c} d$ terms in the order of $100 \mathrm{~s}$ of $S$, the $1 / \eta_{d}$ term in (7a) can be ignored. At high frequencies when $\delta_{1} \ll d_{1}$, surface impedance is completely governed by conductor l's material properties. In between the two extremes, the behavior of $Z_{s}$ is quite complicated and can only be predicted by (4).

\section{Quality Factor of Parallel-Plate Cavity}

From the circuit point of view, increasing loss can lower the $Q$ factor at resonance. To see how it happens, we follow the approaches used in [1]-[4] by rewriting (1) as

$$
Z_{i j}=\sum_{m=0}^{\infty} \sum_{n=0}^{\infty} \frac{N_{m n i} N_{m n j}}{\frac{1}{j \omega L_{m n}}+j \omega C_{m n}+\frac{1}{R_{m n}}}
$$


where

$$
\begin{aligned}
N_{m n i}\left(x_{i}, y_{i} ; d x_{i}, d y_{i}\right)= & \chi_{m n} \cos \left(k_{x m} x_{i}\right) \cos \left(k_{y n} y_{i}\right) \\
& \times \operatorname{sinc}\left(k_{x m} \frac{d x_{i}}{2}\right) \operatorname{sinc}\left(k_{y n} \frac{d y_{i}}{2}\right) \\
N_{m n j}\left(x_{j}, y_{j} ; d x_{j}, d y_{j}\right)= & \chi_{m n} \cos \left(k_{x m} x_{j}\right) \cos \left(k_{y n} y_{j}\right) \\
& \times \operatorname{sinc}\left(k_{x m} \frac{d x_{j}}{2}\right) \operatorname{sinc}\left(k_{y n} \frac{d y_{j}}{2}\right) \\
C_{m n}= & \frac{a b \varepsilon_{d}\left(1+\frac{2 X_{s}}{\omega \mu_{d} h}-\frac{2 R_{s}}{\omega \mu_{d} h} \tan \delta\right)}{h} \\
L_{m n}= & \frac{\mu_{d} h}{a b\left(k_{x m}^{2}+k_{y m}^{2}\right)} \\
R_{m n}= & \frac{a b \omega \varepsilon_{d}\left(\frac{2 R_{s}}{\omega \mu_{d} h}+\tan \delta+\tan \delta \frac{2 X_{s}}{\omega \mu_{d} h}\right)}{h} .
\end{aligned}
$$

The resonant frequency and the $Q$ factor of each mode are given by (9) and (10), shown at the bottom of the page.

It appears that $C_{m n}, R_{m n}, f_{m n}$, and $Q_{m n}$ are all functions of $R_{s}, X_{s}, \tan \delta$, and $h$. For the good conductor and dielectric, the third term inside the parenthesis associated with the $C_{m n}$ and $R_{m n}$ expressions is much smaller than the corresponding first two terms and can be ignored. Similarly, $R_{s} \tan \delta / \eta_{d} h \ll$ $X_{s} / \eta_{d} h$ inside the $f_{m n}$ expression. Thus, as $Z_{s}$ increases, $C_{m n}$ is increased, $R_{m n}$ is decreased, and $f_{m n}$ is slightly decreased from the lossless case. Based on the same reasoning, the third term of both the numerator and denominator of $Q_{m n}$ can be ignored to result in the following approximation:

$$
Q_{m n} \approx \frac{1}{\frac{2 R_{s}}{\omega_{m n} \mu_{d} h}+\tan \delta}=\frac{1}{\frac{1}{Q_{c}}+\frac{1}{Q_{d}}}
$$

where the $Q$ factors due to conductor loss $\left(Q_{c}\right)$ and dielectric loss $\left(Q_{d}\right)$ are given by $Q_{c} \approx \omega_{m n} \mu_{d} h /\left(2 R_{s}\right)$, and $Q_{d} \approx 1 / \tan \delta$.

\section{RESULTS AND DISCUSSIONS}

As mentioned earlier, an effective solution for mitigating power/ground bounce is to reduce board impedance. It can be achieved by adjusting the parameters of the dielectric and the conductor plate. The effect of substrate thickness is considered first. Next, the effect of the magnetic material coating on surface impedance is discussed. Finally, the influence of magnetic material coating on board impedance is analyzed.

\section{A. Effects of Substrate Thickness on Self-/Transfer Impedances and $Q$ Factor}

According to (1), board impedance is proportional to the spacing between the two solid planes. Therefore, reducing the spacing can lower board impedance. Fig. 3(a)-(c) illustrates the self- and transfer impedances and $Q$ factor of a 15.6- by 10.6-cm test board consisting solely of copper power and ground plates of the same thickness $17.5 \mu$ m (i.e., 1/2-oz copper), relative permeability 1 , and conductivity $5.8 \times 10^{7} \mathrm{~S} / \mathrm{m}$, which are separated by a FR-4 substrate with relative permittivity 4.4 and loss tangent 0.019 .

Three different substrate thicknesses of 508, 254, and 101.6 $\mu \mathrm{m}$ (i.e., 20, 10, and 4 mils) are considered. Simulation results clearly demonstrate that the thinnest test board has the lowest resonance impedance peaks and $Q$ factors. As can be seen from (11), the thinner the substrate is, the lower the $Q_{c}$ and $Q_{m n}$ are. In contrast, (8) indicates that thinner substrate produces larger $C_{m n}$, smaller $R_{m n}$ and $L_{m n}$, and, therefore, lower $Z_{i j}$ at all resonant frequencies.

\section{B. Effects of Magnetic Material Coating on Surface Impedance}

As discussed in the previous section, increasing surface impedance can enhance the attenuation constant and decrease the $Q$ factor of the board. Traditionally, manufacturers of PCB use copper as the conductor plate due to its lower dc resistance. However, at high frequency, copper cannot facilitate enough surface impedance to damp natural resonance of the board. The surface impedance of copper is compared with several common magnetic conductors in Fig. 4. The conductivity of copper, iron, cobalt, and nickel are 5.8, 1, 1, and $1.4 \times 10^{7} \mathrm{~S} / \mathrm{m}$, and relative permeability are $1,4000,600$, and 250 , respectively; here, for simplicity, conductivity and permeability are assumed to be frequency independent.

The two lower curves of Fig. 4 are for the two copper plates of thickness 17.5 and $35 \mu \mathrm{m}$. They clearly demonstrate the effects of conductor thickness: 1) A thinner conductor results in larger impedance at low frequencies, and 2) skin effect takes off at a higher frequency with a thinner conductor. When skin effect is fully developed, however, surface impedance depends on the material's conductivity and permeability but not on its thickness. For the four different conductors having the same copper plate thickness of $17.5 \mu \mathrm{m}$, because impedances at low and high frequencies are, respectively, proportional to $1 / \sigma$ and $(\mu / \sigma)^{1 / 2}$, Fig. 4 shows that $Z_{S, \mathrm{Fe}}>Z_{S, \mathrm{Co}}>Z_{S, \mathrm{Ni}}>Z_{S, \mathrm{Cu}}$.

In the previous study, the relative permeability of magnetic material is assumed to be frequency independent. For the real case, however, it will fall off rapidly and result in lower

$$
\begin{aligned}
f_{m n} & =\frac{\sqrt{k_{x m}^{2}+k_{y m}^{2}+\left(\frac{X_{s}}{\eta_{d} h}-\frac{R_{s}}{\eta_{d} h} \tan \delta\right)^{2}}-\left(\frac{X_{s}}{\eta_{d} h}-\frac{R_{s}}{\eta_{d} h} \tan \delta\right)}{2 \pi \sqrt{\mu_{d} \varepsilon_{d}}} \\
Q_{m n} & =\omega_{m n} R_{m n} C_{m n}=\frac{1+\frac{2 X_{s}}{\omega_{m n} \mu_{d} h}-\frac{2 R_{s}}{\omega_{m n} \mu_{d} h} \tan \delta}{\frac{2 R_{s}}{\omega_{m n} \mu_{d} h}+\tan \delta+\frac{2 X_{s}}{\omega_{m n} \mu_{d} h} \tan \delta}
\end{aligned}
$$




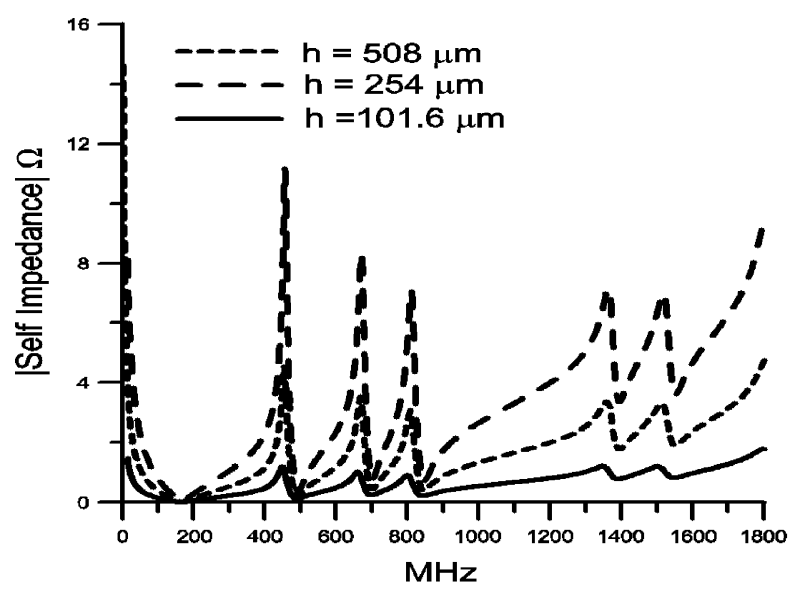

(a)

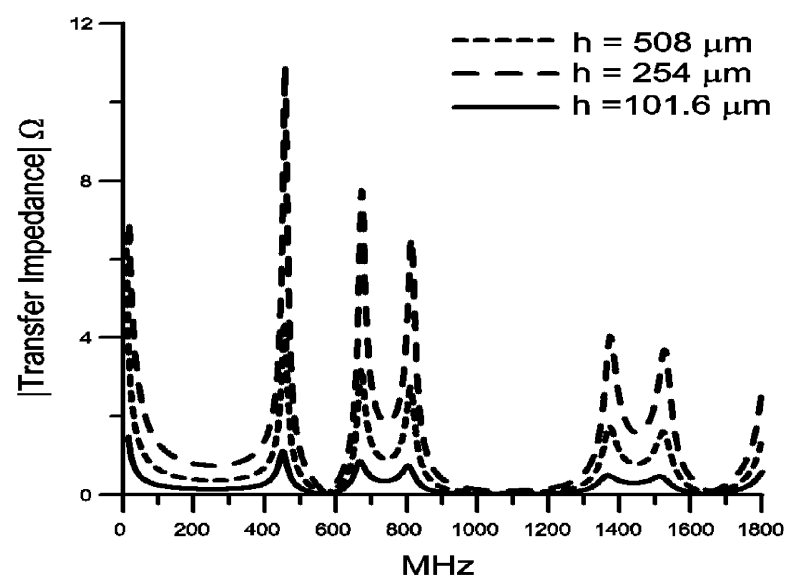

(b)

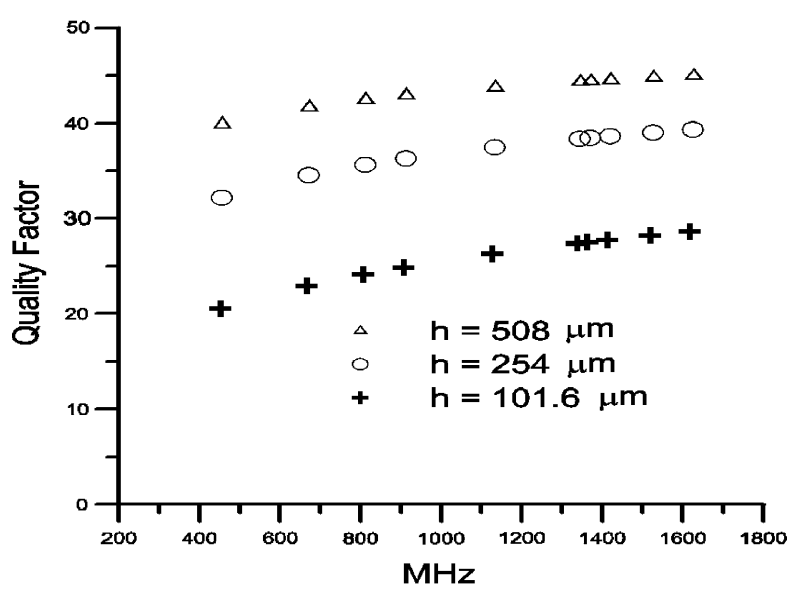

(c)

Fig. 3. (a) $\left|Z_{i i}\right|$, (b) $\left|Z_{i j}\right|$, and (c) $Q_{m n}$ of a $15.6 \times 10.6 \mathrm{~cm}^{2}$ copper powerground plane pair with three different substrate thicknesses. Port $i$ is at $(3.9 \mathrm{~cm}$, $2.65 \mathrm{~cm})$, and port $j$ is at $(11.7 \mathrm{~cm}, 7.95 \mathrm{~cm})$.

surface impedance as frequency increases. To evaluate the influence of frequency-dependent permeability on the self-/transfer impedance of board, an approximate model derived from data reported in [14] for the frequency-dependent permeability of nickel shown in Fig. 5 is considered. Consider the case of a

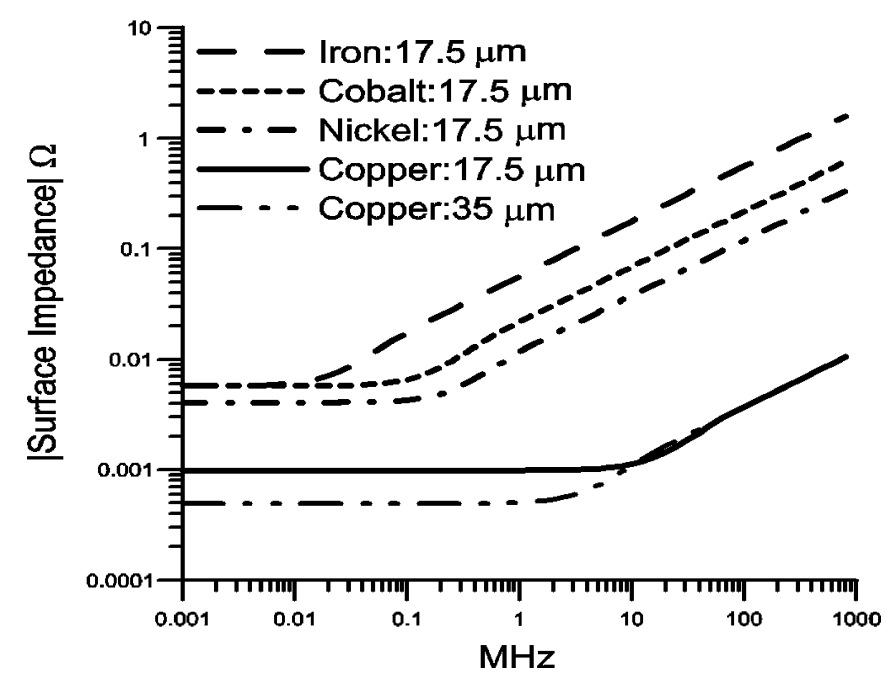

Fig. 4. Surface impedance of copper and several magnetic metals.

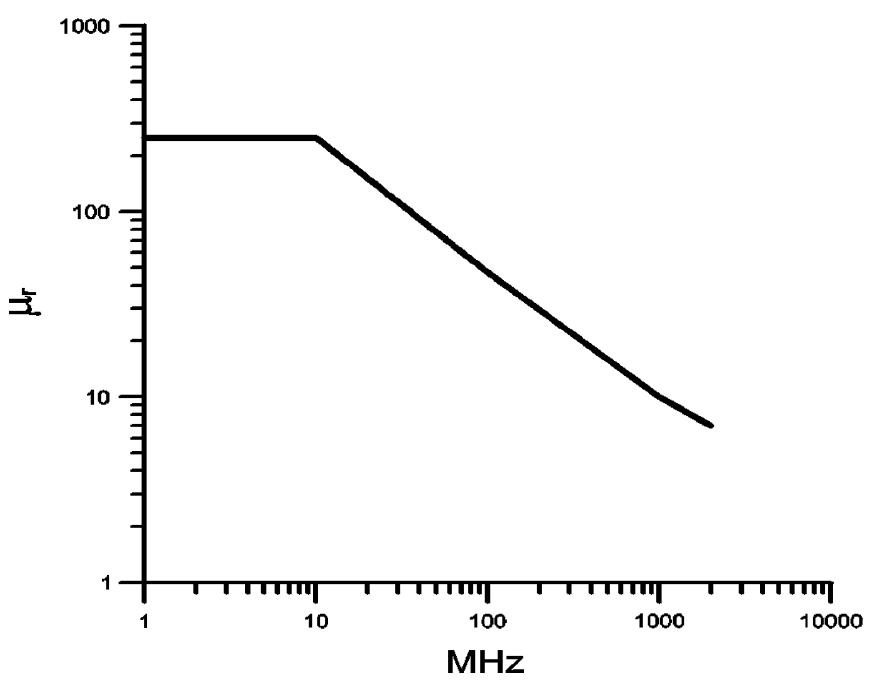

Fig. 5. Frequency-dependent relative permeability of nickel.

17.5- $\mu \mathrm{m}$ copper plate coated with a nickel layer of different thickness: 1, 2, and $4 \mu \mathrm{m}$; the simulation results obtained from (4) for the surface impedance looking from the nickel side are shown in Fig. 6. As predicted by (7), the resulting surface impedance is governed by copper's dc sheet resistance at low frequencies and by nickel's surface impedance at high frequencies. In between, the transition region occurs at a lower (higher) frequency range for a thicker (thinner) nickel coating. As can been seen, a close look at (4) reveals that, because $Z_{S, \mathrm{Ni}}$ is much larger than $Z_{S, \mathrm{Cu}}$, the overall $Z_{S}$ can be simplified as $Z_{S, \mathrm{Cu}}+Z_{S, \mathrm{Ni}} \tanh \left(\gamma_{\mathrm{Ni}} d_{\mathrm{Ni}}\right)$, or $Z_{S, \mathrm{Cu}}+Z_{S, \mathrm{Ni}}\left(\gamma_{\mathrm{Ni}} d_{\mathrm{Ni}}\right)$, at low frequencies. The transition region starts at a frequency when the magnitude of the second term is comparable to that of the first term. Therefore, when the nickel layer thickness increases, the starting frequency of the transition region is decreased. For frequencies above $10 \mathrm{MHz}$, the surface impedance shown in 


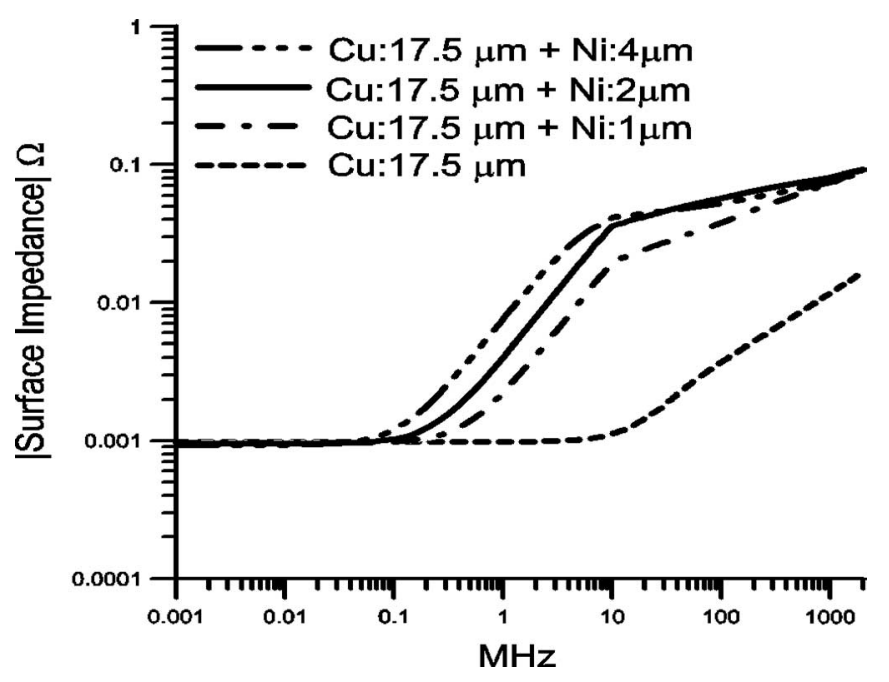

Fig. 6. Surface impedance of nickel-coated copper plate with various thickness of coating.

Fig. 6 increases at a lower rate with increasing frequency due to the reduced relative permeability of nickel.

\section{Effects of Magnetic Material Coating on Self-/Transfer Impedances and $Q$ Factor}

The nickel-coated copper plate whose surface impedance is larger than a pure copper plane is then evaluated for its performance in reducing the self-/transfer impedances of a parallel plane pair. Consider a parallel pair of nickel-coated copper plates of $15.6 \times 10.6 \mathrm{~cm}$, where thickness is $17.5 \mu \mathrm{m}$ for copper, $4 \mu \mathrm{m}$ for nickel, and $101.6 \mu \mathrm{m}, 254 \mu \mathrm{m}$, and $508 \mu \mathrm{m}$ for different FR-4 substrate thickness. The simulation results are shown in Fig. 7.

With Fig. 3, the nickel-coated copper plate has a clear advantage in lowering self-transfer impedances and $Q$ factor when frequency is higher than the board capacitor's self-resonant frequency of about $200 \mathrm{MHz}\left[f_{\mathrm{SR}} \approx 1 / 2 \pi\left(L_{\mathrm{ESL}} C_{00}\right)^{-1 / 2}\right.$, where $L_{\mathrm{ESL}}$ is the equivalent series inductance of the board] because nickel coating results in higher surface impedance and, therefore, a higher attenuation constant. Below the first cavity-resonant frequency (i.e., $f_{10} \approx 458 \mathrm{MHz}$ when losses are ignored), the impedance is mainly contributed by electrostatic capacitance, inductance, and resistance of the board (i.e., $C_{00}, L_{\mathrm{ESL}}$, and $R_{00}$ ). In addition, as evidenced by (9), increased surface impedance also results in a lower board capacitor's self-resonant frequency and cavity-resonant frequencies.

In Fig. 7(a), the self-impedance increases with frequency as $f>f_{\mathrm{SR}}$. It can be explained by reviewing equivalent circuit parameters defined in (8). When the operating frequency approaches each $f_{m n}$, board impedance is mainly contributed by $R_{m n}$ and inductive reactance of the next higherorder mode. Therefore, self-impedance is primarily contributed by the inductive reactance, which increases with increasing frequency because $R_{m n}$ is reduced as surface impedance becomes larger.

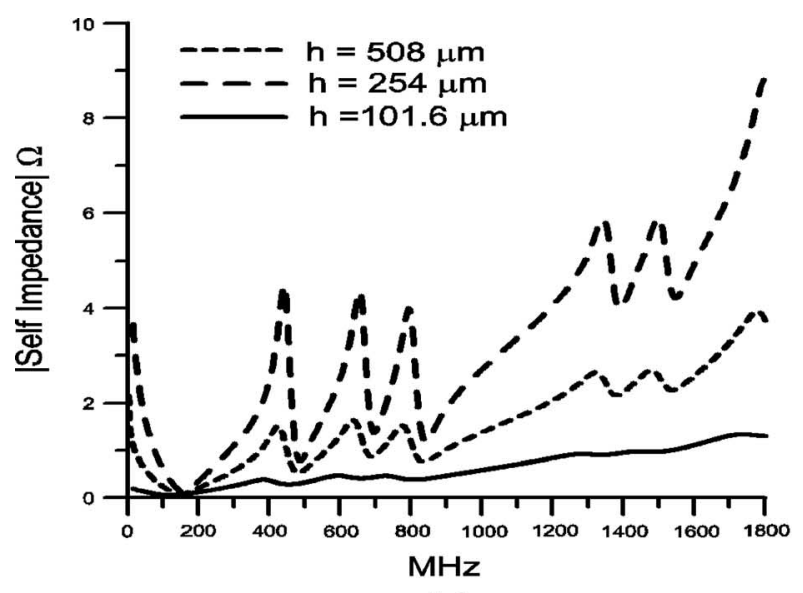

(a)

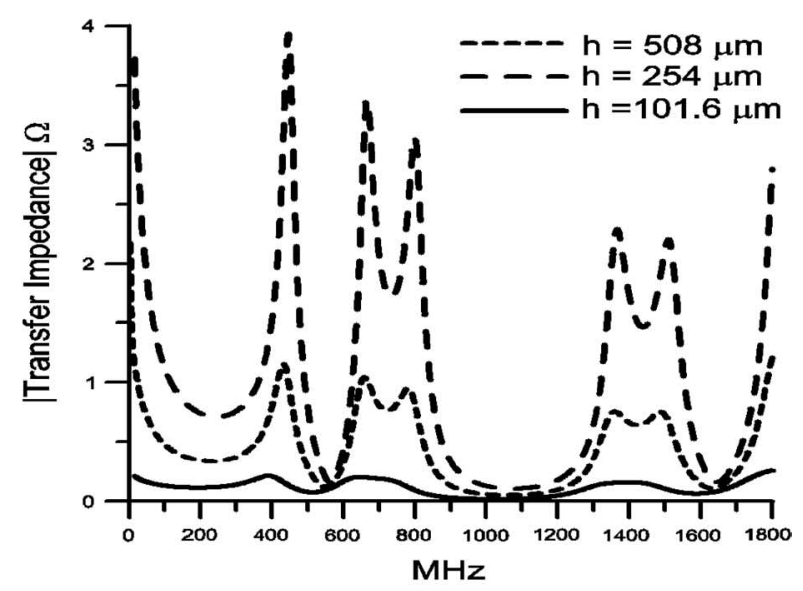

(b)

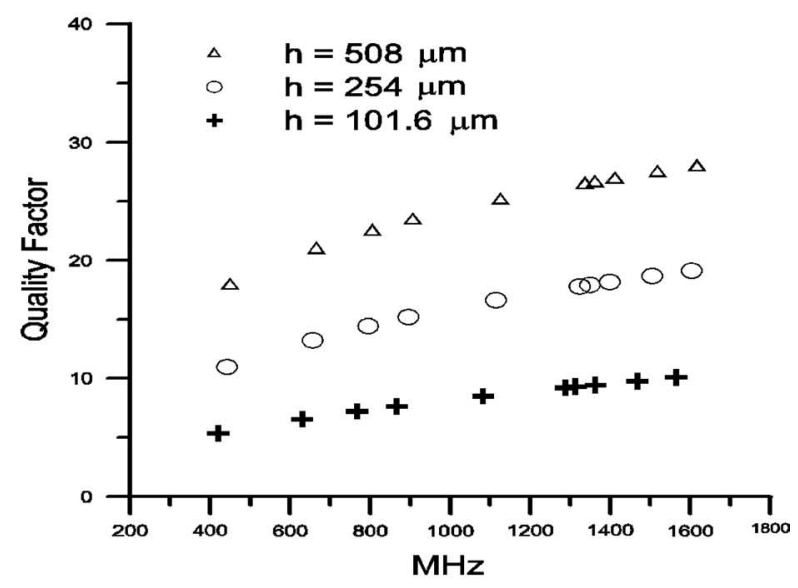

(c)

Fig. 7. (a) $\left|Z_{i i}\right|$, (b) $\left|Z_{i j}\right|$, and (c) $Q_{m n}$ of a $15.6 \times 10.6 \mathrm{~cm}^{2}$ nickel-coated copper power/ground plane pair with three different substrate thicknesses. Port $i$ is at $(3.9 \mathrm{~cm}, 2.65 \mathrm{~cm})$, and port $j$ is at $(11.7 \mathrm{~cm}, 7.95 \mathrm{~cm})$.

\section{CONCLUSION}

A cavity model was used to model the coupling behavior within a parallel plane pair, which behaves like a high $Q$ cavity resonator. Its high-resonance impedance peaks can result in a serious ground bounce problem in high-speed PCBs. An effective approach to mitigate this problem is to reduce board impedance. 
In this paper, the concept of using a thin magnetic material coated over the inside-facing surfaces of conventional copper power and ground plane pair is investigated theoretically. The surface impedance model for the composite two-layer structure is developed and used in the cavity resonator model for the computation of self- and transfer impedances. Example calculations using $4-\mu \mathrm{m}$-thick nickel as the coating material clearly demonstrate its effectiveness in damping high-frequency resonance impedance peaks with negligible effect on the board's dc resistance. The principle behind these is that the use of a thin magnetic material coating causes only a minimal change to the structure's dc resistance (and, therefore, its low-frequency impedance) but a significant increase to its high-frequency surface impedance. This results in much higher high-frequency conductor loss and attenuation constant, which in turn results in much lower resonance impedance peaks and $Q$ factors. The theory developed here is easy to use, applicable for any magnetic material type and thickness, and layout independent.

\section{REFERENCES}

[1] T. Okoshi, Planar Circuits for Microwaves and Lightwaves. Munich, Germany: Springer-Verlag, 1985, pp. 10-42.

[2] M. Xu and T. H. Hubing, "Estimating the power bus impedance of printed circuit boards with embedded capacitance," IEEE Trans. Adv. Packag., vol. 25, no. 3, pp. 424-432, Aug. 2002.

[3] 487489M. Xu, T. H. Hubing, J. Chen, J. L. Drewniak, T. P. Van Doren, and R. E. Dubroff, "Mitigating power bus noise with embedded capacitance in PCB designs," in Proc. IEEE Int. Symp. Electromagn. Compat., Montreal, QC, Canada, Aug. 2001, pp. 487-489.

[4] M. Xu, T. H. Hubing, J. Chen, T. P. Van Doren, J. L. Drewniak, and R. E. DuBroff, "Power-bus decoupling with embedded capacitance in printed circuit board design," IEEE Trans. Electromagn. Compat., vol. 45, no. 1, pp. 22-30, Feb. 2003.

[5] I. Novak, "Reducing simultaneous switching noise and EMI on ground/power planes by dissipative edge termination," IEEE Trans. Adv. Packag., vol. 22, no. 3, pp. 274-283, Aug. 1999.

[6] T. M. Zeeff and T. H. Hubing, "Reducing power bus impedance at resonance with lossy components," in Proc. EPEP Conf., Boston, MA, Oct. 2001.

[7] H. Johnson, "Steel-Plated Power Planes," EDN, Mar. 2002.

[8] Z. L. Wang, O. Wada, Y. Toyota, and R. Koga, "An improved closedform expression for accurate and rapid calculation of power/ground plane impedance of multilayer PCBs," Proc. Symp. Electromagnetic Theory, Toyama, Japan, pp. 17-23, Oct. 2000.
[9] Z. L. Wang, O. Wada, and R. Koga, "Modeling of gapped power bus structures for isolation using cavity modes," IEEE Trans. Electromagn. Compat., vol. 45, no. 3, pp. 10-15, Aug. 2003.

[10] S. Chun, M. Swaminathan, L. D. Smith, J. Srinivasan, Z. Jin, and M. $\mathrm{K}$. Iyer, "Modeling of simultaneous switching noise in high speed systems," IEEE Trans. Adv. Packag., vol. 24, no. 2, pp. 132-142, May 2001.

[11] J. C. Parker Jr., "Via coupling within parallel rectangular planes," IEEE Trans. Electromagn. Compat., vol. 39, no. 1, pp. 17-23, Feb. 1997.

[12] I. Novak, L. M. Noujeim, V. St. Cyr, N. Biunno, A. Patel, G. Korony, and A. Ritter, "Distributed matched bypassing for board-level power distribution networks," IEEE Trans. Adv. Packag., vol. 25, no. 2, pp. 230-243, May 2002.

[13] S. Ramo, J. R. Whinnery, and T. V. Duzer, Fields and Waves in Communication Electronics, 2nd ed., New York: Wiley, 1984, pp. 288-305.

[14] R. M. Bozorth, Ferromagnetism. New York: IEEE Press, 1993, pp. 798 800.

Lin-Kun Wu (S'81-M'81) was born in Hsinchu, Taiwan, R.O.C., in 1958 $\mathrm{He}$ received the M.S. and Ph.D. degrees in electrical and computer engineering from the University of Kansas, Lawrence, in 1982 and 1985, respectively.

From November 1985 to December 1987, he was a Post-Doctoral Research Associate at the Center for Research, Inc., University of Kansas, where he was involved with microwave remote sensing and computational electromagnetics. In 1988, he joined the Department of Communication Engineering, National Chiao Tung University, Hsinchu, Taiwan, where he is currently a Professor. His research interests include computational electromagnetics, biological effects and medial applications of electromagnetic energy, and electromagnetic compatibility.

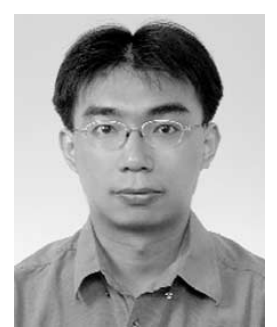

Chih-Hsiung Tseng was born in Kaohsiung, Taiwan, R.O.C., on July 8, 1974. He received the B.S. degree in engineering and system science from National Tsing Hua University, Hsinchu, Taiwan, in 1998 and the M.S degree in communication engineering from the National Chiao Tung University, Hsinchu, in 2000 , where he is currently working toward the Ph.D. degree.

His research interests include computational electromagnetics and the analysis and simulation of ground bounce in high-speed circuit design. 